\title{
A phase III randomized three-arm trial of physical therapist delivered pain coping skills training for patients with total knee arthroplasty: the KASTPain protocol
}

Daniel L Riddle ${ }^{1 *}$, Francis J Keefe ${ }^{2}$, Dennis Ang ${ }^{3}$, Khaled J ${ }^{4}$, Levent Dumenci ${ }^{5}$, Mark P Jensen ${ }^{6}$, Matthew J Bair ${ }^{7}$, Shelby D Reed ${ }^{8}$ and Kurt Kroenke ${ }^{9}$

\begin{abstract}
Background: Approximately 20\% of patients report persistent and disabling pain following total knee arthroplasty (TKA) despite an apparently normally functioning prosthesis. One potential risk factor for unexplained persistent pain is high levels of pain catastrophizing. We designed a three-arm trial to determine if a pain coping skills training program, delivered prior to TKA, effectively reduces function-limiting pain following the procedure in patients with high levels of pain catastrophizing.

Methods/design: The trial will be conducted at four University-based sites in the US. A sample of 402 patients with high levels of pain catastrophizing will be randomly assigned to either a pain coping skills training arm, an arthritis education control arm or usual care. Pain coping skills will be delivered by physical therapists trained and supervised by clinical psychologist experts. Arthritis education will be delivered by nurses trained in the delivery of arthritis-related content. The primary outcome will be change in Western Ontario and McMaster Universities Osteoarthritis Index (WOMAC) Pain scale score 12 months following surgery. A variety of secondary clinical and economic outcomes also will be evaluated.

Discussion: The trial will be conducted at four University-based sites in the US. A sample of 402 patients with high levels of pain catastrophizing will be randomly assigned to either a pain coping skills training arm, an arthritis education control arm or usual care. Pain coping skills will be delivered by physical therapists trained and supervised by clinical psychologist experts. Arthritis education will be delivered by nurses trained in the delivery of arthritis-related content. The primary outcome will be change in Western Ontario and McMaster Universities Osteoarthritis Index (WOMAC) Pain scale score 12 months following surgery. A variety of secondary clinical and economic outcomes also will be evaluated.
\end{abstract}

Trial Registration: NCT01620983

Keywords: Knee, Arthroplasty, Coping skills, Pain, Catastrophizing

\footnotetext{
* Correspondence: dlriddle@vcu.edu

'Departments of Physical Therapy and Orthopaedic Surgery, Virginia

Commonwealth University, Richmond, VA, USA

Full list of author information is available at the end of the article
} 


\section{Background}

Pain is the predominant complaint of patients seeking total knee arthroplasty (TKA) [1-3], a common and generally effective procedure for patients with advanced knee arthritis [1]. Kurtz and colleagues estimated that in 2010 , surgeons would perform over 700,000 TKA procedures in the US and projections suggest 3.5 million TKAs annually by 2030 [4]. Cost data for TKAs also are impressive. Mean procedural and rehabilitation costs per patient, reported in 2006 dollars, were approximately $\$ 20,700$ per primary surgery and $\$ 24,500$ per revision surgery [5] plus significant patient costs incurred over 12 months following surgery [6].

Serious early surgical complications such as pulmonary embolism or joint infection lead to poor outcomes. However, the incidence of these adverse events is very low - approximately $2 \%$ of all surgeries. Failure of the prosthesis is typically a late complication occurring years following the surgery and accounts for approximately 5\% of poor outcomes [7]. The large majority of "poor" outcomes following knee arthroplasty are attributed to disabling pain and impaired function that is not related to early complications or prosthetic loosening. In large patient samples, improvements in pain or function scores have consistently been on the order of $40 \%$ to $60 \%$ relative to baseline, from 6 months to 2 years postoperatively [8-16].

However, some patients respond poorly to the surgery. For example, Puolakka and colleagues found that $36 \%$ of 433 patients reported daily disturbing pain four months or more after surgery $[17,18]$. Hawker et al. reported similar estimates 2 to 7 years following arthroplasty [19]. Only a third of patients report no functional problems following surgery [20] and approximately 20\% report dissatisfaction with their functional ability a year or more after surgery [21]. Functional deficits following surgery are observed in a wide range of activities, with up to $40 \%$ of patients still requiring the use of an assistive device to ambulate [19]. Most recently, Beswick and colleagues reported in a systematic review that $20 \%$ of patients in high quality cohort studies reported persistent function-limiting pain six months or more following TKA [22].

Disabling pain and reduced function is a large and as yet unsolved problem that has a dramatic impact on quality of life and productivity. For example, revision surgery rates are influenced by persistent pain and impaired function. Roberts and colleagues conducted a survival analysis of 4,400 patients with knee arthroplasty and found that 15 years following surgery, a total of 239 knees required revision and up to $35 \%$ were for unexplained pain. Extrapolating to current estimates, as many as $35 \%$ of 55,000 revision arthroplasty surgeries in the US in 2010 may be attributable to unexplained persistent pain and subsequent poor function [23].
A barrier to improving postoperative outcomes is that traditionally, knee arthroplasty has been presumed to be a highly effective procedure. TKA perioperative protocols have historically not incorporated routine screening for patients at-risk for post-surgery persistent pain or compromised function because this area has not been scientifically investigated. This culture has strong potential for change, however, because recent research has begun to acknowledge that unexplained poor outcomes occur [22]. Predictors of these poor outcomes following knee arthroplasty have been identified [24-27]. Among the most consistent and powerful psychological predictors of poor outcome following knee arthroplasty is pain catastrophizing [17,26,28-32]. Individuals who catastrophize tend to ruminate about pain, magnify the threat value of pain and feel helpless when dealing with pain $[33,34]$.

Additional impetus to address the issue of persistent pain was proposed by the National Institutes of Health (NIH). An NIH consensus panel was convened to review existing evidence regarding the use of knee arthroplasty surgery and to make recommendations for future research to improve the care for these patients [1]. The panel placed high priority on research examining the impact of perioperative interventions for these patients. Our trial will specifically target this research need. If a high-quality trial demonstrates that pain coping skills training is successful at improving outcomes for at-risk patients with poor pain coping, and is cost effective, current clinical practice paradigms could be significantly improved.

The primary aim of our study is to assess the efficacy of a physical therapist-delivered pain coping skills training program in reducing knee pain and improving function. Our two primary hypotheses are that in patients scheduled for knee arthroplasty and with comorbid pain catastrophizing: (1) Pain coping skills training is more effective than arthritis education in decreasing knee pain during functional activities, and (2) Pain coping skills training is more effective than usual care in decreasing knee pain during functional activities. We also will examine two sets of secondary hypotheses: (1) Pain coping skills training is more effective than arthritis education or usual care in improving self-reported function, physical performance, pain intensity, pain catastrophizing, and patient global ratings of improvement and (2) Pain coping skills training will reduce direct medical costs and indirect (i.e. patient time) costs relative to arthritis education and usual care. We hypothesize that, in addition to accounting for costs associated with pain coping skills training, the intervention will be cost saving or costeffective relative to arthritis care education and usual care as measured by the incremental cost per qualityadjusted life-year. We will also determine if treatment 
benefits are mediated by changes in pain catastrophizing: We hypothesize that treatment-related changes in pain catastrophizing will mediate treatment-related improvements in pain and self-reported function during recovery.

\section{Method/design}

\section{Study design}

The KASTPain Trial is a three-arm randomized trial (see Figure 1) funded by the National Institute of Arthritis, Musculoskeletal and Skin Diseases/National Institutes of Health (1UM1AR062800). The investigators, research assistants assessing outcomes, and patients assigned to two of the three study arms will be blinded to group assignment. Potential subjects will be informed that they will be randomly assigned to one of two different educational treatments or usual care. They will likely not know whether they will be receiving the treatment with the hypothesized key ingredient (pain coping skills training). The protocol conforms to the CONSORT guidelines for nonpharmacologic interventions [35]. Human subjects approval for the study has been obtained from the Virginia Commonwealth University Institutional Review Board (HM14326).

\section{Study population}

We will recruit 402 patients who consult with participating orthopaedic surgeons in one of four sites for TKA. These sites are: (1) Virginia Commonwealth University in Richmond, Virginia; (2) Southern Illinois University in Springfield, Illinois; (3) Duke University in Durham, North Carolina; and (4) Wake Forest University, WinstonSalem North Carolina. To be eligible for participation all patients must: (1) be able to read and speak English and provide informed consent; (2) be 45 years or older; (3) have a diagnosis of osteoarthritis as determined by the patients' orthopaedic surgeons; (4) be scheduled for an elective unilateral total knee arthroplasty no sooner than 2 weeks or later than 8 weeks from the time of recruitment; and

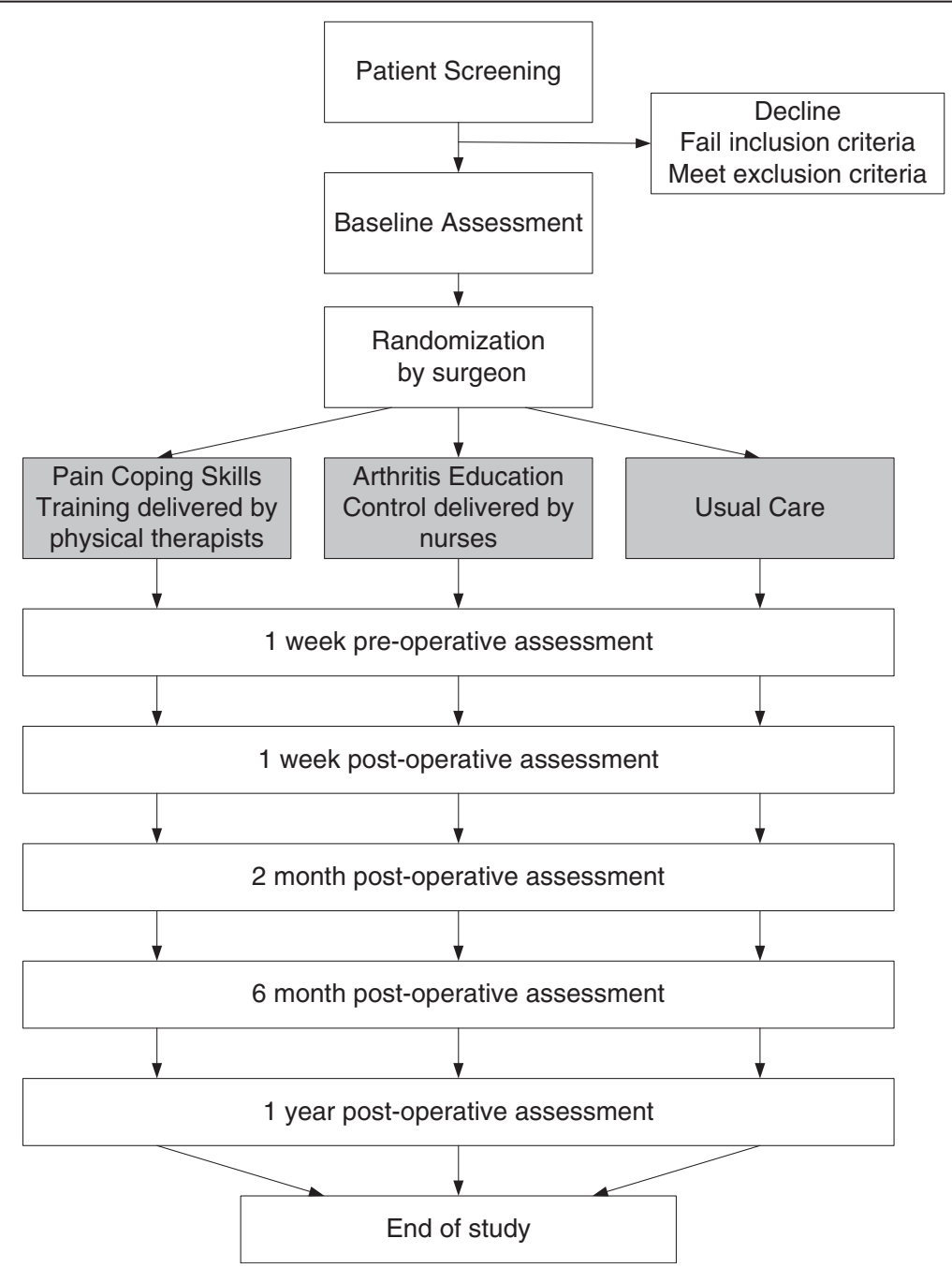

Figure 1 Legend: The figure illustrates the flow of subjects through the trial. 
(5) score of $\geq 16$ on the Pain Catastrophizing Scale and $\geq 5$ on the WOMAC Pain scale. Scores of 16 or greater on the Pain Catastrophizing Scale suggest at least a moderately severe pain catastrophizing while scores of $\geq 5$ on the WOMAC Pain Scale suggests greater than minor function limiting pain. Patients will be excluded if they: (1) are scheduled for revision arthroplasty surgery; (2) are unable to or decline consent for study participation; (3) have a self-reported diagnosis of inflammatory arthritis (i.e. rheumatoid arthritis, psoriatic arthritis, systemic lupus erythematosis, ankylosing spondylitis); (4) have TKA scheduled because of a fracture, malignancy or an infection; (5) are scheduled for bilateral TKA; (6) are scheduled for unicompartmental arthroplasty; (7) report plans to undergo hip or knee arthroplasty within one year after current TKA; (8) underwent contralateral knee arthroplasty surgery or hip arthroplasty surgery within 1 year of currently planned surgery; or (9) score 20 or higher on the Patient Health Questionnaire-8 (PHQ-8) depression screener which indicates likely severe depression [36,37]. Pregnancy is an "automatic" exclusion because women who are pregnant are excluded by their physicians from TKA.

\section{Procedures}

All patients scheduled for TKA with orthopaedic surgeons who conduct at least 50 TKAs per year at one of the four university-based study sites will potentially be eligible for study. We will only recruit surgeons who conduct $\geq 50$ TKAs per year because of the potential influence of TKA volume on outcome [38]. Following an opt-out period, each patient will be contacted by phone to assess for eligibility after providing verbal consent for screening. All patients enrolled in the study will attend an in-person session to read and sign the consent form and complete preoperative data collection procedures. In addition, all patients will complete a series of performance-based measures which include a 6-minute walk test [39] and the short physical performance battery [40], which includes a series of tests of walking speed, balance and strength.

Two in-person data collection sessions will occur for each patient, one at the baseline visit and the other at 1 year following TKA. In addition, staff that are blinded to group assignment will contact patients by phone at 4 different time points; 1 week prior to and 1 week following TKA, 2 months, 6 months following TKA (see Figure 1) to collect outcome data. Key measures obtained at each time point will be the Pain Catastrophizing Scale, the WOMAC Scale, and a knee pain rating scale [42].

\section{Randomization and allocation concealment}

All eligible subjects will be randomized following baseline data collection into the pain coping skills arm, the arthritis education control arm or the usual care arm. Patients will be blinded to study hypotheses and will be informed that the study is examining the potential benefits of two approaches to improve outcomes as compared to usual care. The study statistician will prepare a random numbers table to permit randomization in permuted block sizes of 3 and 6, stratified by surgeon. To conceal randomization, assignment will occur via the web interface only after all baseline data are collected. The physical therapists delivering the pain coping skills and the nurses delivering the arthritis education cannot be blinded.

\section{Interventions}

Participants randomized to one of the two treatment arms will receive eight 1-hour sessions of one-on-one instruction to be delivered over a 2-month period beginning approximately 2 weeks prior to surgery and ending 6 weeks following surgery. The first session will be delivered in-person and subsequent sessions will be delivered via the telephone. Telephone-based behavioral interventions for pain management have been shown to be effective $[42,43]$

\section{Pain coping skills}

The 8-session pain coping skills training (CST) will be delivered over a 2-month period by physical therapists. This number of sessions of CST has been shown to be effective in several behavioral trials [44-50]. Study physical therapists must have practiced for at least 2 years and reported experience in the treatment of patients with TKA.

The (CST) protocol will: (1) provide a rationale for the coping skills intervention; (2) train patients in cognitive restructuring as well as a variety of skills that provide patients with opportunities to observe the impact of coping skills on changes in negative pain-related cognitions typical of pain catastrophizing (i.e. thoughts related to pain rumination, pain magnification, and helplessness in the face of pain); and (3) provide training in strategies for enhancing maintenance of gain following treatment. Melzack and Wall's gate control model of pain will be used to help patients reconceptualize their pain and emphasize their own abilities to control pain. The gate control model highlights the role that thoughts, feelings, and behaviors can play in influencing the transmission of noxious signals from the periphery to the brain [51]. Training in pain coping skills will be described as a way of changing thoughts, feelings, and behaviors that contribute to pain.

Using techniques drawn from cognitive therapy, patients will be taught how to identify irrational, maladaptive, and catastrophic pain-related thoughts and to replace these with alternative, rational, reassuring and 
adaptive thoughts [52]. Self-instructional training will be used to teach patients how to use calming selfstatements as a way of coping with pain flares [53]. Activity-rest cycling and pleasant activity scheduling [54-56] will be used to help patients increase their activity level and observe the resultant impact on their painrelated cognitions. Activity-rest cycling teaches patients to target activities they tend to overdo (e.g. prolonged standing or walking while shopping) and learn to break these activities into periods of moderate activity (e.g. 30 minutes of shopping) followed by a limited rest break (e.g. 5 minutes of rest). Over time, the goal is to help patients raise their activity level by increasing the length of their activity and decreasing the length of their rest periods. In pleasant activity scheduling, patients learn how to identify activities they enjoy doing (e.g. reading, doing hobbies, and visiting friends) or that give them a sense of mastery (learning how to do something new such as typing or a new language) and then set and record weekly activity goals.

Patients also will be trained in three attention diversion methods that can be used to alter negative pain related cognitions: relaxation, imagery, and distraction. Progressive relaxation training [57] will help patients learn to concentrate on muscle tension signals and use them as cues to relax. Patients will be taught how to use pleasant imagery as a way to alter their pain-related thought patterns and foster relaxation [58]. Distraction training will involve training in how to focus on physical stimuli (e.g. a photograph or picture of a nature scene) or auditory stimuli (e.g. listening to music) when experiencing increased pain [58]. Using relapse prevention methods, each patient will develop a written maintenance plan that includes the list of pain coping skills learned during the study, potential high risk situations, early warning signs of setbacks, and plans about how the patient might apply these skills in dealing with future setbacks and challenges. Table 1 highlights the key elements of the CST protocol.

\section{Arthritis education}

Patients randomly assigned to the arthritis education arm will receive detailed information from a registered

$\begin{aligned} & \text { Table } \mathbf{1} \text { Components of the pain coping skills } \\
& \text { intervention }\end{aligned}$
\begin{tabular}{ll}
\hline Training objective & Coping skill training methods \\
\hline $\begin{array}{l}\text { Altering Cognitions to Change } \\
\text { Pain Catastrophizing }\end{array}$ & $\begin{array}{l}\text { Cognitive Restructuring, } \\
\text { Self-Instructional Training }\end{array}$ \\
$\begin{array}{l}\text { Altering Activity Patterns To } \\
\text { Change Pain Catastrophizing }\end{array}$ & Activity-Rest Cycling, Goal Setting \\
$\begin{array}{l}\text { Using Attention Diversion to } \\
\text { Change Pain Catastrophizing }\end{array}$ & $\begin{array}{l}\text { Relaxation Training, } \\
\text { Imagery, Distraction }\end{array}$ \\
Enhancing Maintenance & Relapse Prevention Training \\
\hline
\end{tabular}

or licensed practical nurse educator about osteoarthritis and its treatment. The arthritis education intervention will control for participation in a trial, time and clinician attention. The arthritis education sessions will use a presentation and discussion format similar to that originally described by Lorig for arthritis education [59-62]. Figures and discussion sessions will present information on the nature of arthritis, the post-operative course of knee arthroplasty, treatment of osteoarthritis, the role of exercise, joint protection and making future treatment decisions.

This general approach to an arthritis education comparison condition has been used successfully in many behavioral studies and in several trials conducted by Keefe and Jensen [44-50,62]. An education comparison condition is a credible treatment and allows us to test whether the "pain coping" component of the experimental intervention is the specific treatment element that reduces pain and improves function over that seen in patients who receive a similar dose of time and attention from a health professional, but no training in pain coping skills.

\section{Usual care}

We have added a "usual care" group to determine real world effects of pain coping skills. Patients in the usual care group will only receive care that they would have routinely received had they not been entered in the study. All patients in this group will undergo the same data collection procedures as patients in the other two treatment arms with the exception of the eight treatment sessions.

\section{Co-interventions}

Patients are routinely prescribed medications for pain control and are referred for physical therapy following TKA. Data regarding medication and extent of rehabilitation therapies will be tracked throughout the study period and will be adjusted for, if necessary, in the analyses.

\section{Treatment adherence and fidelity}

Because multiple study sites will participate and increase the risk of low fidelity due to differing approaches in applying the interventions, the research team will take a number of steps to ensure that the treatment protocols for CST as well as arthritis education are delivered uniformly by all treatment providers involved in the study. First, all pain coping skills training will be delivered by physical therapists with at least 2 years of clinical experience including the treatment of patients following TKA. Second, all physical therapists will receive coping skills training in a 2-day workshop delivered by clinical psychologist experts at Duke University. Experienced nurse 
educators will all be trained in 2-day workshops by DLR, a physical therapist with 30 years of experience and research in arthritis and rehabilitation, to provide the arthritis education. Third, all physical therapists and nurse educators will be provided with detailed treatment manuals and the treatment strategies will be taught through didactic instruction, illustrations of techniques from model cases, and role-play of common scenarios. Fourth, we will institute several "best practices" to enhance and monitor treatment fidelity of the pain coping skills training and arthritis education which include: (1) careful attention to the study design; (2) intensive training; (3) role playing of treatment skills enactment during training; and (4) on-line documentation of treatment delivery. All sessions for both groups will be audiotaped and supervisors or investigators will review tapes periodically for each physical therapist and nurse educator during the study. Remedial training will be provided for those clinicians who deviate from established protocol.

\section{Outcomes}

The primary outcome measure and endpoint will be the WOMAC Pain score measured 1 year following knee arthroplasty [63-67]. The WOMAC has been studied extensively and its scales have been shown to be reliable and valid for quantifying the extent of both pain and disability in patients undergoing knee arthroplasty $[16,68,69]$. Secondary outcome measures will be the WOMAC Disability scale, Pain Catastrophizing Scale (PCS) [33,70,71], a verbal pain rating scale [72,73], and a patient global rating of change scale measured on a numerical rating scale from -5 (vastly worse) to +5 (completely recovered) [74-76]. All outcome measures are summarized in Table 2. Outcomes will be measured pre-operatively, 2 months, 6 months and 1 year post-surgery. To assess for potential mediating effects of pain catastrophizing on outcomes, the PCS and WOMAC scales will be administered one week prior to and 1 week following TKA. Two performance-based measures, the 6-minute walk test $[39,77]$ and the Short Physical Performance Battery will be assessed at baseline and 1 year post-surgery [40].

\section{Power considerations and data analysis}

The primary endpoint will be the WOMAC Pain score at 1 year. Changes of 2 or more points in the 20-point WOMAC Pain scale indicate clinically important differences in pain-related function between individual patients [78-81]. We powered the KASTPain study to detect a difference of at least 2 points between the mean pain scores in the pain coping skills group and the arthritis education group [82].

Using a two-sided, two-group t-test of differences in means with alpha set at 0.05 and assuming the
Table 2 Summary of primary and secondary outcome and cost measure

\begin{tabular}{ll}
\hline Measure & Specific instrument \\
\hline Primary outcome Measure & WOMAC Pain Scale \\
(3.1 Likert version)
\end{tabular}

intervention difference minus the arthritis education control difference is at least 2 WOMAC Pain points, a sample size of 107 in each group will provide $91 \%$ power to detect this difference, assuming that the common standard deviation is 4.34 (based on pilot work). This corresponds to a moderate effect size of 0.46 which is consistent with the effect of other behavioral interventions for knee arthritis [83-85]. This sample size also provides $80 \%$ power to detect a $20 \%$ difference between groups in the proportion of patients with a $50 \%$ or greater improvements in WOMAC pain relative to baseline scores $[9,10,12-15,86]$. This effect is equivalent to an odds ratio of 2.25 and a number needed to treat (NNT) of 5 [87].

The required sample size is 321 (107 patients per arm) for the planned three-arm trial. Based on our pilot study, we expect $5 \%$ attrition due to early drop-out resulting from cancelled surgery. Patients who undergo surgery and drop out due to lack of interest, unrelated medical illness or loss of follow-up will be included in the intentto-treat analysis. Our previous work with similar types of patients suggests that loss to follow-up will likely be approximately $20 \%$ one year following surgery [14,30]. Therefore, 402 patients (134 patients per study arm) will need to be enrolled in the trial. The accrual and retention numbers will be monitored during the study to assure that sample size estimates are reached. Stratification by surgeon is expected to reduce outcome variability and thereby increase power. 
Intention to treat (ITT) will be the primary approach for all analyses. Because we expect that some patients will provide consent for our study but may opt out of surgery for a variety of reasons, we will compare the ITT analysis to the results for patients who actually undergo surgery (i.e., attrition analysis). For the primary analysis, the effect of treatment will be assessed using linear mixed models with time as a repeated factor. The model will account for correlation over time within participants, correlations within surgeons, and baseline covariates. Surgical approach, complications, medication and physical therapy use will be assessed for potential effects. Restricted maximum likelihood method (REML), which uses all available data, will be used to estimate the linear mixed model. REML is the default option in multiple software packages for mixed models including SAS and Mplus. Wothke has shown that no other missing data handling method, regardless of missing data mechanism, performs better [88].

Estimates of the effect of pain coping skills training will be obtained by constructing linear contrasts to compare the outcome at each of the key time points (baseline, 2 months, 6 months and 1 year) between the pain coping skills group and the two control groups, with adjustment for the other variables. Analyses of the secondary outcome variables will be conducted using a similar approach. For the analyses using dichotomous outcomes of $\geq 50 \%$ improvement at 6 months and 12 months, generalized linear models with logistic regression will be used to compare the proportion of patients with $\geq 50 \%$ improvement after adjustment for covariates.

As an additional secondary analysis, we also will examine the effects of potential moderators. Moderators are patient characteristics that predict treatment effects [89]. Additional psychosocial issues may influence treatment effects in knee arthroplasty. Potential moderators for patients with knee arthroplasty who may particularly benefit from pain coping skills training are the following: treatment expectations prior to surgery [90-92], self efficacy $[93,94]$, extent of social support $[9,95]$ and depression [28]. Because our study is not powered to test for these potential moderators, they will be assessed in the context of hypothesis generation rather than hypothesis testing and only for the primary outcome of WOMAC Pain at 1 year. This analysis will be performed by including a two-way Moderator X Treatment group interaction term in the mixed models analyses. We will assess whether these variables independently predict those patients who are more likely to respond to pain coping skills training versus arthritis education or usual care. Pain coping skills training emphasizes pain coping strategies, unlike usual care or arthritis education and because of this emphasis, we suspect that coping strategies training will be particularly effective in patients with adverse psychosocial characteristics. Knowing whether any of these potential moderators actually predict response to treatment may aid in better identifying individuals who are more likely to respond to the intervention.

\section{Economic evaluation}

\section{Medical resource use and total costs}

We will also compare medical resource use and mean total costs incurred over the one-year follow-up period in the KASTPain study between patients randomized to coping skills training vs. arthritis education vs. usual care. Counts of medical resource use will include inpatient stays, outpatient visits to physicians, physical therapists and other providers, and days of pain medication. Sources for unit costs assigned to medical resource use will include average Medicare payments for inpatient care and outpatient services and average wholesale prices published in the Red Book for medications. The TEAM-HF Costing Tool will be used to estimate intervention costs by accounting for providers' time spent delivering the study intervention [96]. Total costs will consist of direct medical costs associated with the study interventions and costs associated with TKA, rehabilitation, complications and pain management as well as patients' time costs associated with receipt of the study interventions. Total costs, from the societal perspective, will consist of direct medical costs, intervention-related costs and indirect costs. From the health care system (or payer) perspective, indirect costs will be excluded. Sensitivity analyses will be performed to evaluate the impact of scaling up the intervention (i.e. more patients per session; fixed costs allocated over more patients) and methodological choices for cost assignment [97,98].

\section{Cost-Effectiveness analysis}

A cost-effectiveness analysis will also be performed. Incremental cost-effectiveness ratios (ICER) will be calculated as the difference in the mean costs per patient between study arms divided by the difference in estimated quality-adjusted life-years (QALYS). Mean costs will be estimated as described above. QALYs will be estimated using patient-level utility estimates derived from the EQ-5D, administered at baseline, 2 months, 6 months and 1 year [99]. Because the study interventions represent fixed-, one-time costs while the benefits of the interventions may last beyond the one year follow-up period in the KASTPain study, we will conduct sensitivity analyses that extrapolate differences in utilities measured at the end of follow-up over 3, 5, and 10 years, assuming that the interventions do not differentially impact survival. If we observe statistically significant differences in costs (not including costs for the intervention or initial surgery) between treatment arms at 12 months, we will extrapolate treatment-specific cost estimates over 
3,5 , and 10 years, consistent with the time period for QALYs. We will evaluate uncertainty by estimating 95\% CIs for estimates of costs, QALYs and measures of costeffectiveness (i.e. ICERs or net health benefits [100]) using nonparametric bootstrapping.

\section{Discussion}

Our study will be the first to examine the efficacy of a pain coping skills training intervention delivered by physical therapists to patients at risk for poor outcome following orthopaedic surgery. The use of physical therapists as interventionists is innovative because the intervention is traditionally delivered by clinical psychologists. However, given the limited availability of clinical psychologists and the large volume of TKA procedures, physical therapists routinely treat patients prior to and following TKA and are optimally positioned to provide coping skills training.

Previous work by our group [30] and others $[28,29,32,101]$ has identified a TKA patient subgroup at risk for poor outcome. Patients with high levels of pain catastrophizing have been consistently shown to have a higher rate of persistent pain and compromised function compared to non-catastrophizers [28,102,103]. Current clinical paradigms do not discuss formal identification of patients at-risk for poor outcome due to ineffective pain coping $[104,105]$ nor do these paradigms address the use of perioperative interventions to reduce poor outcome risk. Our trial will provide high-quality evidence to potentially challenge this practice paradigm. If the KASTPain intervention is effective, the research will provide strong evidence to consider augmenting this traditional approach with a scalable intervention that could improve pain and functioning for thousands of patients with TKA who are at high risk for poor outcomes.

\section{Abbreviations \\ TKA: Total knee arthroplasty; WOMAC: Western Ontario and McMaster Universities Osteoarthritis Index.; NIH: National Institutes of Health; PHQ-8: Patient Health Questionnaire-8; CST: Pain coping skills training; PCS: Pain Catastrophizing Scale; NNT: Number needed to treat; ITT: Intention to treat; REML: Restricted maximum likelihood method; ICER: Incremental cost effectiveness ratio; QALYS: Quality adjusted life years.}

\section{Competing interests}

Drs. Riddle, Keefe Ang, Saleh, Dumenci, Jensen, Bair, Reed and Kroenke have no competing interests to declare.

\section{Authors' contributions}

All authors participated in the conception and design of the protocol and helped to draft the manuscript. All authors read and approved the final manuscript.

\section{Acknowledgements}

The study was funded by the National Institute of Arthritis, Musculoskeletal and Skin Diseases/National Institutes of Health (1UM1AR062800).

\section{Author details}

'Departments of Physical Therapy and Orthopaedic Surgery, Virginia Commonwealth University, Richmond, VA, USA. ${ }^{2}$ Department of Psychiatry and Behavioral Sciences, Duke Pain Prevention and Treatment Research Program, Duke University, Durham, NC, USA. ${ }^{3}$ Section of Rheumatology, Wake Forest University, Winston-Salem, NC, USA. ${ }^{4}$ Department of Orthopaedic Surgery, Southern Illinois University, Springfield, IL, USA. ${ }^{5}$ Department of Social and Behavioral Health, Virginia Commonwealth University, Richmond, VA, USA. 'Department of Rehabilitation Medicine, University of Washington, Harborview Medical Center, Seattle, WA, USA ${ }^{7}$ Roudebush VA Medical Center of Excellence on Implementing EvidenceBased Practice, Indianapolis, IN 46202, USA. ${ }^{8}$ Duke Clinical Research Institute and Department of Medicine, Duke University School of Medicine, Durham, NC 27715, USA. 'Department of Medicine, Indiana University, Indianapolis, IN, USA.

Received: 25 July 2012 Accepted: 15 August 2012

Published: 20 August 2012

\section{References}

1. NIH Consensus Statement on total knee replacement December 8-10, 2003. J Bone Joint Surg Am 2004, 86-A:1328-1335.

2. Lingard EA, Sledge $C B$, Learmonth ID: Patient expectations regarding total knee arthroplasty: differences among the United States, United kingdom, and Australia. J Bone Joint Surg Am 2006, 88:1201-1207.

3. Noble PC, Conditt MA, Cook KF, Mathis KB: The John Insall Award: Patient expectations affect satisfaction with total knee arthroplasty. Clin Orthop Relat Res 2006, 452:35-43.

4. Kurtz S, Ong K, Lau E, Mowat F, Halpern M: Projections of primary and revision hip and knee arthroplasty in the United States from 2005 to 2030. J Bone Joint Surg Am 2007, 89:780-785.

5. Losina E, Walensky RP, Kessler CL, Emrani PS, Reichmann WM, Wright EA Holt HL, Solomon DH, Yelin E, Paltiel AD, et al: Cost-effectiveness of total knee arthroplasty in the United States: patient risk and hospital volume. Arch Intern Med 2009, 169:1113-1121.

6. Marshall DA, Wasylak T, Khong H, Parker RD, Faris PD, Frank C: Measuring the value of total hip and knee arthroplasty: considering costs over the continuum of care. Clin Orthop Relat Res 2012, 470:1065-1072.

7. Manley M, Ong K, Lau E, Kurtz SM: Total knee arthroplasty survivorship in the United States Medicare population: effect of hospital and surgeon procedure volume. J Arthroplasty 2009, 24:1061-1067.

8. Brander VA, Stulberg SD, Adams AD, Harden RN, Bruehl S, Stanos SP, Houle T: Predicting total knee replacement pain: a prospective, observational study. Clin Orthop Relat Res 2003, 416:27-36.

9. Escobar A, Quintana JM, Bilbao A, Azkarate J, Guenaga Jl, Arenaza JC, Gutierrez LF: Effect of patient characteristics on reported outcomes after total knee replacement. Rheumatology (Oxford) 2007, 46:112-119.

10. Fortin PR, Clarke AE, Joseph L, Liang MH, Tanzer M, Ferland D, Phillips C, Partridge AJ, Belisle $P$, Fossel AH, et al: Outcomes of total hip and knee replacement: preoperative functional status predicts outcomes at six months after surgery. Arthritis Rheum 1999, 42:1722-1728.

11. Fortin PR, Penrod JR, Clarke AE, St-Pierre Y, Joseph L, Belisle P, Liang MH, Ferland D, Phillips CB, Mahomed N, et al: Timing of total joint replacement affects clinical outcomes among patients with osteoarthritis of the hip or knee. Arthritis Rheum 2002, 46:3327-3330.

12. Heck DA, Robinson RL, Partridge CM, Lubitz RM, Freund DA: Patient outcomes after knee replacement. Clin Orthop Relat Res 1998, 356:93-110.

13. Fitzgerald JD, Orav EJ, Lee TH, Marcantonio ER, Poss R, Goldman L, Mangione CM: Patient quality of life during the 12 months following joint replacement surgery. Arthritis Rheum 2004, 51:100-109.

14. Lingard EA, Katz JN, Wright EA, Sledge CB: Predicting the outcome of total knee arthroplasty. J Bone Joint Surg Am 2004, 86-A:2179-2186.

15. Bachmeier CJ, March LM, Cross MJ, Lapsley HM, Tribe KL, Courtenay BG, Brooks PM: A comparison of outcomes in osteoarthritis patients undergoing total hip and knee replacement surgery. Osteoarthritis Cartilage 2001, 9:137-146.

16. Escobar A, Quintana JM, Bilbao A, Arostegui I, Lafuente I, Vidaurreta I: Responsiveness and clinically important differences for the WOMAC and SF-36 after total knee replacement. Osteoarthritis Cartilage 2007, 15:273-280.

17. Baron RM, Kenny DA: The moderator-mediator variable distinction in social psychological research: conceptual, strategic, and statistical considerations. J Pers Soc Psychol 1986, 51:1173-1182. 
18. Puolakka PA, Rorarius MG, Roviola M, Puolakka TJ, Nordhausen K, Lindgren L: Persistent pain following knee arthroplasty. Eur J Anaesthesiol 2010, 27:455-460

19. Hawker G, Wright J, Coyte P, Paul J, Dittus R, Croxford R, Katz B, Bombardier C, Heck D, Freund D: Health-related quality of life after knee replacement. J Bone Joint Surg Am 1998, 80:163-173.

20. Wright RJ, Sledge CB, Poss R, Ewald FC, Walsh ME, Lingard EA: Patient-reported outcome and survivorship after Kinemax total knee arthroplasty. J Bone Joint Surg Am 2004, 86-A:2464-2470.

21. Jones CA, Voaklander DC, Johnston DW, Suarez-Almazor ME: Health related quality of life outcomes after total hip and knee arthroplasties in a community based population. J Rheumatol 2000, 27:1745-1752.

22. Beswick AD, Wylde V, Gooberman-Hill R, Blom A, Dieppe P: What proportion of patients report long-term pain after total hip or knee replacement for osteoarthritis? A systematic review of prospective studies in unselected patients. BMJ Open 2012, 2:e000435.

23. Roberts VI, Esler CN, Harper WM: A 15-year follow-up study of 4606 primary total knee replacements. J Bone Joint Surg Br 2007, 89:1452-1456.

24. Ayers DC, Franklin PD, Trief PM, Ploutz-Snyder R, Freund D: Psychological attributes of preoperative total joint replacement patients: implications for optimal physical outcome. J Arthroplasty 2004, 19:125-130.

25. Ayers DC, Franklin PD, Ploutz-Snyder R, Boisvert CB: Total knee replacement outcome and coexisting physical and emotional illness. Clin Orthop Relat Res 2005, 440:157-161.

26. Ethgen O, Bruyere O, Richy F, Dardennes C, Reginster JY: Health-related quality of life in total hip and total knee arthroplasty. A qualitative and systematic review of the literature. J Bone Joint Surg Am 2004, 86-A:963-974.

27. Jones DL, Westby MD, Greidanus N, Johanson NA, Krebs DE, Robbins L, Rooks DS, Brander V: Update on hip and knee arthroplasty: current state of evidence. Arthritis Rheum 2005, 53:772-780.

28. Edwards RR, Haythornthwaite JA, Smith MT, Klick B, Katz JN: Catastrophizing and depressive symptoms as prospective predictors of outcomes following total knee replacement. Pain Res Manag 2009, 14:307-311.

29. Forsythe ME, Dunbar MJ, Hennigar AW, Sullivan MJ, Gross M: Prospective relation between catastrophizing and residual pain following knee arthroplasty: two-year follow-up. Pain Res Manag 2008 13:335-341.

30. Riddle DL, Wade JB, Jiranek WA, Kong X: Preoperative Pain Catastrophizing Predicts Pain Outcome after Knee Arthroplasty. Clin Orthop Relat Res 2009, 468:798-806

31. Roth ML, Tripp DA, Harrison MH, Sullivan M, Carson P: Demographic and psychosocial predictors of acute perioperative pain for total knee arthroplasty. Pain Res Manag 2007, 12:185-194.

32. Sullivan M, Tanzer M, Stanish W, Fallaha M, Keefe FJ, Simmonds M, Dunbar M: Psychological determinants of problematic outcomes following Total Knee Arthroplasty. Pain 2009, 143:123-129.

33. Sullivan MJL, Bishop S, Pivik J: The Pain Catastrophizing Scale: Development and Validation. Psych Assessment 1995, 7:524-532.

34. Turner JA, Aaron LA: Pain-related catastrophizing: what is it? Clin J Pain 2001, 17:65-71.

35. Boutron I, Moher D, Altman DG, Schulz KF, Ravaud P: Extending the CONSORT statement to randomized trials of nonpharmacologic treatment: explanation and elaboration. Ann Intern Med 2008, 148:295-309.

36. Kroenke K, Spitzer RL, Williams JB: The PHQ-9: validity of a brief depression severity measure. J Gen Intern Med 2001, 16:606-613.

37. Kroenke K, Strine TW, Spitzer RL, Williams JB, Berry JT, Mokdad AH: The PHQ-8 as a measure of current depression in the general population. J Affect Disord 2009, 114:163-173.

38. Katz JN, Barrett J, Mahomed NN, Baron JA, Wright RJ, Losina E: Association between hospital and surgeon procedure volume and the outcomes of total knee replacement. J Bone Joint Surg Am 2004, 86-A:1909-1916.

39. Guyatt GH, Sullivan MJ, Thompson PJ, Fallen EL, Pugsley SO, Taylor DW, Berman LB: The 6-minute walk: a new measure of exercise capacity in patients with chronic heart failure. Can Med Assoc J 1985, 132:919-923.
40. Guralnik JM, Simonsick EM, Ferrucci L, Glynn RJ, Berkman LF, Blazer DG, Scherr PA, Wallace RB: A short physical performance battery assessing lower extremity function: association with self-reported disability and prediction of mortality and nursing home admission. J Gerontol 1994, 49:M85-M94.

41. Jensen MP, Turner JA, Romano JM, Fisher LD: Comparative reliability and validity of chronic pain intensity measures. Pain 1999, 83:157-162.

42. Allen KD, Oddone EZ, Coffman CJ, Datta SK, Juntilla KA, Lindquist JH, Walker TA, Weinberger M, Bosworth HB: Telephone-based selfmanagement of osteoarthritis: A randomized trial. Ann Intern Med 2010, 153:570-579.

43. McBeth J, Prescott G, Scotland G, Lovell K, Keeley P, Hannaford P, McNamee P, Symmons DP, Woby S, Gkazinou C, et al: Cognitive behavior therapy, exercise, or both for treating chronic widespread pain. Arch Intern Med 2012, 172:48-57.

44. Dalton JA, Keefe FJ, Carlson J, Youngblood R: Tailoring cognitive-behavioral treatment for cancer pain. Pain Manag Nurs 2004, 5:3-18.

45. Keefe FJ, Caldwell DS, Baucom D, Salley A, Robinson E, Timmons K, Beaupre P, Weisberg J, Helms M: Spouse-assisted coping skills training in the management of osteoarthritic knee pain. Arthritis Care Res 1996, 9:279-291.

46. Keefe FJ, Caldwell DS, Baucom D, Salley A, Robinson E, Timmons K, Beaupre P, Weisberg J, Helms M: Spouse-assisted coping skills training in the management of knee pain in osteoarthritis: long-term followup results. Arthritis Care Res 1999, 12:101-111.

47. Keefe FJ, Blumenthal J, Baucom D, Affleck G, Waugh R, Caldwell DS, Beaupre P, Kashikar-Zuck S, Wright K, Egert J, et al: Effects of spouseassisted coping skills training and exercise training in patients with osteoarthritic knee pain: a randomized controlled study. Pain 2004, 110:539-549.

48. Keefe FJ, Anderson T, Lumley M, Caldwell D, Stainbrook D, McKee D, Waters SJ, Connelly M, Affleck G, Pope MS, et al: A randomized, controlled trial of emotional disclosure in rheumatoid arthritis: Can clinician assistance enhance the effects? Pain 2008, 137:167-172

49. Martire LM, Schulz R, Keefe FJ, Starz TW, Osial TA Jr, Dew MA, Reynolds CF III: Feasibility of a dyadic intervention for management of osteoarthritis: a pilot study with older patients and their spousal caregivers. Aging Ment Health. 2003, 7:53-60.

50. Turner JA, Jensen MP: Efficacy of cognitive therapy for chronic low back pain. Pain 1993, 52:169-177.

51. Melzack R: Recent concepts of pain. J Med 1982, 13:147-160.

52. Beck AT, Rush AJ, Shaw BF, Emery G: Cognitive Therapy and Depression. New York: Guilford Press; 1979

53. Turk DC, Meichenbaum D, Genest M: Pain and Behavioral Medicine: A cognitive-behavior perspective. New York: Guilford; 1983

54. Houpt JL, Keefe FJ, Snipes MT: The Clinical Specialty Unit: the use of the psychiatry inpatient unit to treat chronic pain syndromes. Gen Hosp Psychiatry. 1984, 6:65-70

55. Keefe FJ: Behavioral assessment and treatment of chronic pain: current status and future directions. J Consult Clin Psychol 1982, 50:896-911.

56. Lewinson PM: The behavioral study and treatment of depression. In Progress in Behavior Modification. Edited by Hersen M, Eisler RM, Miller PM; 1975:19-65.

57. Surwit RS: Progressive Relaxation Training Manual. Duke: University Medicial Center; 1979.

58. McCaul KD, Malott JM: Distraction and coping with pain. Psychol Bull 1984, 95:516-533.

59. Lorig K: Patient education: treatment or nice extra. Br J Rheumatol 1995, 34:703-704.

60. Lorig K: Arthritis self-help course. HMO Pract 1995, 9:60-61

61. Lorig K, Gonzalez VM, Laurent DD, Morgan L, Laris BA: Arthritis self-management program variations: three studies. Arthritis Care Res 1998, 11:448-454

62. Keefe FJ, Caldwell D, Williams DA, Gil KM, Mitchell D: Pain coping skills training in the management of osteoarthritic knee pain: A comparative study. Behav Ther 1990, 21:49-62

63. Bellamy N, Buchanan WW: A preliminary evaluation of the dimensionality and clinical importance of pain and disability in osteoarthritis of the hip and knee. Clin Rheumatol 1986, 5:231-241. 
64. Bellamy N, Buchanan WW, Goldsmith CH, Campbell J, Stitt LW: Validation study of WOMAC: a health status instrument for measuring clinically important patient relevant outcomes to antirheumatic drug therapy in patients with osteoarthritis of the hip or knee. J Rheumatol 1988, 15:1833-1840

65. Bellamy N: Pain assessment in osteoarthritis: experience with the WOMAC osteoarthritis index. Semin Arthritis Rheum 1989, 18:14-17.

66. Bellamy N, Campbell J, Stevens J, Pilch L, Stewart C, Mahmood Z: Validation study of a computerized version of the Western Ontario and McMaster Universities VA3.0 Osteoarthritis Index. J Rheumatol 1997, 24:2413-2415.

67. Bellamy N, Kirwan J, Boers M, Brooks P, Strand V, Tugwell P, Altman R, Brandt K, Dougados M, Lequesne M: Recommendations for a core set of outcome measures for future phase III clinical trials in knee, hip, and hand osteoarthritis. Consensus development at OMERACT III. J Rheumatol 1997, 24:799-802.

68. Hawker G, Melfi C, Paul J, Green R, Bombardier C: Comparison of a generic (SF-36) and a disease specific (WOMAC) (Western Ontario and McMaster Universities Osteoarthritis Index) instrument in the measurement of outcomes after knee replacement surgery. J Rheumatol 1995, 22:1193-1196

69. McConnell S, Kolopack P, Davis AM: The Western Ontario and McMaster Universities Osteoarthritis Index (WOMAC): a review of its utility and measurement properties. Arthritis Rheum 2001, 45:453-461.

70. Chibnall JT, Tait RC: Confirmatory factor analysis of the Pain Catastrophizing Scale in African American and Caucasian Workers' Compensation claimants with low back injuries. Pain 2005, 113:369-375.

71. Osman A, Barrios FX, Gutierrez PM, Kopper BA, Merrifield T, Grittmann L: The Pain Catastrophizing Scale: further psychometric evaluation with adult samples. J Behav Med 2000, 23:351-365.

72. Dworkin RH, Turk DC, Farrar JT, Haythornthwaite JA, Jensen MP, Katz NP, Kerns RD, Stucki G, Allen RR, Bellamy N, et al: Core outcome measures for chronic pain clinical trials: IMMPACT recommendations. Pain 2005 113:9-19.

73. Jensen MP, Karoly P, Braver S: The measurement of clinical pain intensity: a comparison of six methods. Pain 1986, 27:117-126.

74. Kamper SJ, Maher CG, Mackay G: Global rating of change scales: a review of strengths and weaknesses and considerations for design. J Man Manip Ther 2009, 17:163-170.

75. Kamper SJ, Ostelo RW, Knol DL, Maher CG, de Vet HC, Hancock MJ: Global Perceived Effect scales provided reliable assessments of health transition in people with musculoskeletal disorders, but ratings are strongly influenced by current status. J Clin Epidemiol 2010.

76. Hancock MJ, Maher CG, Latimer J, McLachlan AJ, Cooper CW, Day RO, Spindler MF, McAuley JH: Assessment of diclofenac or spinal manipulative therapy, or both, in addition to recommended first-line treatment for acute low back pain: a randomised controlled trial. Lancet 2007 370:1638-1643.

77. Kennedy DM, Stratford PW, Wessel J, Gollish JD, Penney D: Assessing stability and change of four performance measures: a longitudinal study evaluating outcome following total hip and knee arthroplasty. BMC Musculoskelet Disord 2005, 6:3.

78. Angst F, Aeschlimann A, Steiner W, Stucki G: Responsiveness of the WOMAC osteoarthritis index as compared with the SF-36 in patients with osteoarthritis of the legs undergoing a comprehensive rehabilitation intervention. Ann Rheum Dis 2001, 60:834-840.

79. Angst F, Aeschlimann A, Stucki G: Smallest detectable and minimal clinically important differences of rehabilitation intervention with their implications for required sample sizes using WOMAC and SF-36 quality of life measurement instruments in patients with osteoarthritis of the lower extremities. Arthritis Rheum 2001, 45:384-391.

80. Angst F, Aeschlimann A, Michel BA, Stucki G: Minimal clinically important rehabilitation effects in patients with osteoarthritis of the lower extremities. J Rheumatol 2002, 29:131-138.

81. Ehrich EW, Davies GM, Watson DJ, Bolognese JA, Seidenberg BC, Bellamy N: Minimal perceptible clinical improvement with the Western Ontario and McMaster Universities osteoarthritis index questionnaire and global assessments in patients with osteoarthritis. J Rheumatol 2000, 27:2635-2641.

82. Riddle DL, Keefe FJ, Nay WT, McKee D, Attarian DE, Jensen MP: Pain Coping Skills Training for Patients With Elevated Pain Catastrophizing Who Are
Scheduled for Knee Arthroplasty: A Quasi-Experimental Study. Arch Phys Med Rehabil 2011, 92:859-865.

83. Heuts PH: de BR, Drietelaar M, Aretz K, Hopman-Rock M, Bastiaenen $\mathrm{CH}$ Metsemakers JF, van WC, van SO. Self-management in osteoarthritis of hip or knee: a randomized clinical trial in a primary healthcare setting. J Rheumatol 2005, 32:543-549.

84. Hurley MV, Walsh NE, Mitchell HL, Pimm TJ, Patel A, Williamson E, Jones RH, Dieppe PA, Reeves BC: Clinical effectiveness of a rehabilitation program integrating exercise, self-management, and active coping strategies for chronic knee pain: a cluster randomized trial. Arthritis Rheum 2007, 57:1211-1219.

85. Nunez M, Nunez E, Segur JM, Macule F, Quinto L, Hernandez MV, Vilalta C: The effect of an educational program to improve health-related quality of life in patients with osteoarthritis on waiting list for total knee replacement: a randomized study. Osteoarthritis Cartilage 2006, 14:279-285.

86. Brander V, Gondek S, Martin E, Stulberg SD: Pain and depression influence outcome 5 years after knee replacement surgery. Clin Orthop Relat Res 2007, 464:21-26.

87. McQuay HJ, Moore RA: Using numerical results from systematic reviews in clinical practice. Ann Intern Med 1997, 126:712-720.

88. Wothke W: Longitudinal and multigroup modeling with missing data. In Modeling longitudinal and multilevel data: Practical issues, applied approaches, and specific examples. Edited by Little TDSKUBJ. Mahwah, New Jersey: Lawrence Erlbaum Associates; 2000:219-40.

89. Kraemer HC, Wilson GT, Fairburn CG, Agras WS: Mediators and moderators of treatment effects in randomized clinical trials. Arch Gen Psychiatry 2002, 59:877-883.

90. Gandhi R, Davey JR, Mahomed NN: Predicting patient dissatisfaction following joint replacement surgery. J Rheumatol 2008, 35:2415-2418

91. Gandhi R, Davey JR, Mahomed N: Patient expectations predict greater pain relief with joint arthroplasty. J Arthroplasty 2009, 24:716-721.

92. Mahomed NN, Liang MH, Cook EF, Daltroy LH, Fortin PR, Fossel AH, Katz JN: The importance of patient expectations in predicting functional outcomes after total joint arthroplasty. J Rheumatol 2002, 29:1273-1279.

93. Khan CM, lida M, Stephens MA, Fekete EM, Druley JA, Greene KA: Spousal support following knee surgery: roles of self-efficacy and perceived emotional responsiveness. Rehabil Psychol 2009, 54:28-32

94. Wylde V, Dieppe P, Hewlett S, Learmonth ID: Total knee replacement: is it really an effective procedure for all? Knee 2007, 14:417-423.

95. Gandhi R, Razak F, Davey JR, Rampersaud YR, Mahomed NN: Effect of sex and living arrangement on the timing and outcome of joint replacement surgery. Can J Surg 2010, 53:37-41.

96. Reed SD, Li Y, Kamble S, Polsky D, Graham FL, Bowers MT, Samsa GP, Paul S, Schulman KA, Whellan DJ, et al: Introduction of the Tools for Economic Analysis of Patient Management Interventions in Heart Failure Costing Tool: a user-friendly spreadsheet program to estimate costs of providing patient-centered interventions. Circ Cardiovasc Qual Outcomes 2012, 5:113-119.

97. Reed SD, Li Y, Oddone EZ, Neary AM, Orr MM, Grubber JM, Graham FL, Olsen MK, Svetkey LP, Dolor RJ, et al: Economic evaluation of home blood pressure monitoring with or without telephonic behavioral selfmanagement in patients with hypertension. Am J Hypertens 2010, 23:142-148.

98. Reed SD, Whellan DJ, Li Y, Friedman JY, Ellis SJ, Pina IL, Reed SD, Whellan DJ, Li Y, Friedman JY, Ellis SJ, Pina IL, Settles SJ, Vidson-Ray L, Johnson JL, Cooper LS, et al: Economic evaluation of the HF-ACTION (Heart Failure: A Controlled Trial Investigating Outcomes of Exercise Training) randomized controlled trial: an exercise training study of patients with chronic heart failure. Circ Cardiovasc Qual Outcomes 2010, 3:374-381.

99. Herdman M, Gudex C, Lloyd A, Janssen M, Kind P, Parkin D, Bonsel G, Badia $X$ : Development and preliminary testing of the new five-level version of EQ-5D (EQ-5D-5L). Qual Life Res 2011, 20:1727-1736.

100. Stinnett AA, Mullahy J: Net health benefits: a new framework for the analysis of uncertainty in cost-effectiveness analysis. Med Decis Making 1998, 18:S68-S80. 
101. Gandhi R, Tsvetkov D, Dhottar H, Davey JR, Mahomed NN: Quantifying the pain experience in hip and knee osteoarthritis. Pain Res Manag 2010, 15:224-228.

102. Edwards RR, Bingham CO III, Bathon J, Haythornthwaite JA: Catastrophizing and pain in arthritis, fibromyalgia, and other rheumatic diseases. Arthritis Rheum 2006, 55:325-332.

103. Edwards RR, Cahalan C, Mensing G, Smith M, Haythornthwaite JA: Pain, catastrophizing, and depression in the rheumatic diseases. Nat Rev Rheumatol 2011, 7:216-224

104. NIH Consensus Panel: NIH Consensus Statement on total knee replacement December 8-10, 2003. J Bone Joint Surg Am 2004, 86-A:1328-1335.

105. Cross WW III, Saleh KJ, Wilt TJ, Kane RL: Agreement about indications for total knee arthroplasty. Clin Orthop Relat Res 2006, 446:34-39.

doi:10.1186/1471-2474-13-149

Cite this article as: Riddle et al: A phase III randomized three-arm trial of physical therapist delivered pain coping skills training for patients with total knee arthroplasty: the KASTPain protocol. BMC Musculoskeletal Disorders 2012 13:149.

\section{Submit your next manuscript to BioMed Central and take full advantage of:}

- Convenient online submission

- Thorough peer review

- No space constraints or color figure charges

- Immediate publication on acceptance

- Inclusion in PubMed, CAS, Scopus and Google Scholar

- Research which is freely available for redistribution 